\title{
A Formal Analysis of Bluetooth Device Discovery ${ }^{\star}$
}

\author{
Marie Duflot, Marta Kwiatkowska, Gethin Norman and David Parker \\ School of Computer Science, University of Birmingham, Birmingham, B15 2TT, UK
}

\begin{abstract}
This paper presents a formal analysis of the device discovery phase of the Bluetooth wireless communication protocol. The performance of this process is the result of a complex interaction between several devices, some of which exhibit random behaviour. We use probabilistic model checking and, in particular, the tool PRISM to compute the best and worst case expected time for device discovery. We illustrate the utility of performing an exhaustive, low-level analysis to produce exact results in contrast to simulation techniques, where additional probabilistic assumptions must be made. We demonstrate an example of how seemingly innocuous assumptions can lead to incorrect performance estimations. We also analyse the effectiveness of improvements made between versions 1.1 and 1.2 of the Bluetooth specification.
\end{abstract}

\section{Introduction}

The use of formal methods for the verification and analysis of systems is becoming more and more prevalent in industry. Increasingly, these techniques are being applied not just to ascertain correctness, but also to analyse quantitative properties such as performance and reliability. In this paper, we demonstrate the applicability of an automated formal verification technique called probabilistic model checking to an analysis of the performance of the Bluetooth protocol.

Bluetooth is a wireless telecommunication technology, aimed in particular at low-power devices communicating over short distances. It is becoming increasingly prominent in devices such as mobile phones, PDAs and laptop computers. To cope with interference, Bluetooth is based on frequency-hopping technology. This means that, before any communication can take place, an initialisation procedure must be carried out, comprising discovery of devices in the vicinity and then exchange of information to synchronise hopping sequences. From a user's point of view, this process affects both the waiting-time and the power usage. Hence, our analysis focuses on this aspect of the protocol.

As will be demonstrated shortly, the time required for completion of the Bluetooth initialisation process is the result of a non-trivial interaction between two devices, motivating the need for a formal, automated analysis. Furthermore, it includes a randomised back-off procedure to resolve contention between devices, and an effective analysis thus needs to be able to reason about the stochastic

\footnotetext{
* Supported by FORWARD and EPSRC grants GR/S46727 and GR/S11107.
} 
nature of the system. We use probabilistic model checking and in particular the tool PRISM. This process involves construction of a formal probabilistic model from a high-level description of the system, followed by calculation of one or more probabilistic properties, formally expressed in probabilistic temporal logic.

In contrast to approaches based on discrete-event simulation, for which analyses of Bluetooth have already been attempted, formal approaches such as probabilistic model checking involve an exhaustive analysis. We construct the full model and use it to compute actual performance values, rather than derive estimations from a large number of simulations. As we will show later, this means we can examine worst-case behaviour rather than simplifying with probabilistic assumptions and generating average values. Furthermore, we can identify precisely the situations that lead to these worst-case scenarios. We will also give an example of a situation where making additional probabilistic assumptions leads to inaccuracies in the performance results obtained.

\section{Probabilistic Model Checking and PRISM}

Probabilistic model checking is an automated technique for the formal verification of systems that exhibit stochastic behaviour. It is based on the construction and analysis of a mathematical model of the system, usually from a specification in some high-level description language. This model generally comprises a set of states, representing all the possible configurations of the system, the transitions that can occur between these states, and information about when and with what probability each transition will occur.

In this paper, the modelling formalism we use is discrete-time Markov chains (DTMCs), where time is modelled as discrete steps and the probability of making each transition is given by a discrete probability distribution. Other model types commonly used are continuous-time Markov chains (CTMCs), Markov decision processes (MDPs), and probabilistic timed automata (PTAs); see 9] for more detailed information about these. We use the probabilistic model checking tool PRISM [72]. This allows construction of models via specification in a highlevel description language, based on the parallel composition of several modules described in a guarded command notation. We will illustrate the workings of this language in more detail later in the paper.

Models constructed in PRISM are analysed by formally specifying properties in temporal logic. This allows reasoning, for example, about "the probability of shutdown occurring within 24 hours" or "the long-run probability that the system is stable". In addition, by assigning real-valued costs (or, conversely, rewards) to states and transitions of the model, we can also reason about, for example, "expected time" or "expected power consumption". PRISM automatically ascertains values for these properties by performing probabilistic model checking, which includes both graph-based analysis and numerical computation. For the case of DTMCs, the latter usually constitutes solving a linear equation system of size equal to the number of states in the model, for which PRISM uses iterative numerical solution methods. 
A significant amount of work has gone into the development of efficient, symbolic implementation techniques for numerical computation. These use data structures based on binary decision diagrams (BDDs) to allow compact storage and manipulation of extremely large models. We rely heavily on this efficiency for the case study presented in this paper.

The PRISM tool has already been successfully used to perform analysis of and identify interesting behaviour in a wide range of case studies. This includes the study of "quality of service" properties for components of real-time probabilistic communication protocols such as IEEE 1394 FireWire, IEEE 802.3 CSMA/CD, Zeroconf and IEEE 802.11 wireless LANs. It has also been used to verify randomised distributed algorithms for leader election, self-stabilisation, mutual exclusion, consensus and Byzantine agreement, and probabilistic security protocols for anonymity, fair exchange and contract signing. Finally, PRISM has been applied to analysing the performance and reliability of many different types of applications: dynamic power management schemes, NAND multiplexing for nanotechnology, queueing systems, computer networks, manufacturing processes and embedded systems. The reader is invited to consult the web site [2] for detailed information and corresponding publications about all of these.

\section{Device Discovery in Bluetooth}

Bluetooth is a short-range, low-power, open standard for implementing wireless personal area networks. Since it uses the unlicensed $2.4 \mathrm{GHz}$ Industry Scientific and Medical band (a set of frequencies almost globally available), there is a potential problem of interference from other devices using this band. To resolve this, Bluetooth uses a frequency hopping scheme, where devices alternate rapidly among the 79 available frequencies in a pseudo-random fashion.

In order to communicate, Bluetooth devices organise themselves into small networks called piconets, comprising one master and up to 7 slave devices, in which the frequency hopping sequences are synchronised and controlled by the master. In this paper, we focus on the issue of piconet creation, the performance of which is crucial because no communication between devices can occur until it is complete. It also has considerably higher power consumption than other parts of the protocol [6], prevents existing device connections from operating and may cause interference to other nearby piconets.

Piconet formation has two steps: firstly, the inquiry process, where a master device discovers neighbouring slave devices; and secondly, the page process, where connections between them are established. During the first step, information about slave clock times is exchanged for the purposes of synchronisation. This can be used during the second step, which is hence much faster. We therefore concentrate on the inquiry process. We now describe in more detail the procedure executed by an inquiring device (a master trying to discover slaves) and a scanning device (a potential slave device who wants to be discovered). 


\subsection{The Inquiring Device}

An inquiring device attempts to detect potential slaves in the proximity by broadcasting inquiry packets on a previously agreed sequence of 32 of the 79 available frequencies and scanning for replies. This process continues until some specified bound on the number of replies received or the total time is exceeded.

Like all Bluetooth devices, the inquiring device has a 28 bit free-running clock, which ticks every $312.5 \mu$ s. On two consecutive $312.5 \mu$ s time slots, it sends on two sequential frequencies. During the next two time slots, the device scans for a reply on these same two frequencies, i.e. each scan occurs $625 \mu$ s after the corresponding send (in fact, a $10 \mu \mathrm{s}$ margin is added to the start and end of the scan in case replying devices are not completely synchronised). The device now proceeds to send and scan on the next pair of frequencies in the same fashion. This procedure is illustrated in Figure 1 .

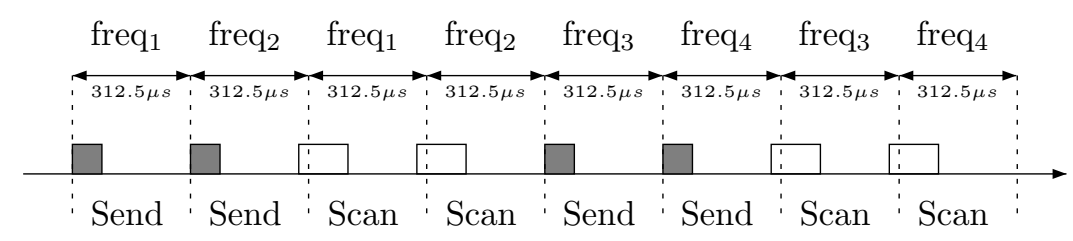

Fig. 1. Timing of the inquiring device's behaviour

The 32 frequencies used for the inquiry procedure are split into two trains, $\mathrm{A}$ and $\mathrm{B}$, of 16 frequencies each. The sequence in which the master device sends and scans on these frequencies is determined by its 28 bit clock, denoted $C L K$, according to the following formula:

$$
\text { freq }=\left[C L K_{16-12}+k+\left(C L K_{4-2,0}-C L K_{16-12}\right) \bmod 16\right] \bmod 32
$$

where $C L K_{i-j}$ denotes bits $i, \ldots, j$ of $C L K$, and $k$ is an offset to select whether train A or B is used. The inquiring device swaps between train A and B every 2.56 seconds: the time to send and scan on 16 frequencies is $10 \mathrm{~ms}$ and each train is repeated 256 times. Furthermore, every 1.28 seconds (every time the 12th bit of $C L K$ changes), a frequency is swapped between train $\mathrm{A}$ and $\mathrm{B}$. The whole list of frequencies is shown in Figure 2. Each line of this table is repeated 128 times, taking 1.28 seconds. To simplify the presentation, we have assumed $k=1$ for train $\mathrm{A}$ and $k=17$ for train $\mathrm{B}$, i.e. initially trains $\mathrm{A}$ and $\mathrm{B}$ comprise frequencies $1 \ldots 16$ and $17 \ldots 32$, respectively.

\subsection{The Scanning Device}

Bluetooth devices that want to be discovered enter the inquiry scan substate and periodically scan for inquiry packets on the same 32 frequencies that the inquiring device is transmitting on. To ensure that the frequencies used eventually coincide and that messages are successfully received, the hopping rate of scanning devices 


\begin{tabular}{|c|c|c|c|c|c|c|c|c|c|c|c|c|c|c|c|}
\hline 1 & 2 & 3 & 4 & 5 & 6 & 7 & 8 & 9 & 10 & 11 & 12 & 13 & 14 & 15 & 16 \\
\hline 17 & 2 & 3 & 4 & 5 & 6 & 7 & 8 & 9 & 10 & 11 & 12 & 13 & 14 & 15 & 16 \\
\hline 1 & 2 & 19 & 20 & 21 & 22 & 23 & 24 & 25 & 26 & 27 & 28 & 29 & 30 & 31 & 32 \\
\hline 1 & 2 & 3 & 20 & 21 & 22 & 23 & 24 & 25 & 26 & 27 & 28 & 29 & 30 & 31 & 32 \\
\hline 17 & 18 & 19 & 20 & 5 & 6 & 7 & 8 & 9 & 10 & 11 & 12 & 13 & 14 & 15 & 16 \\
\hline 17 & 18 & 19 & 20 & 21 & 6 & 7 & 8 & 9 & 10 & 11 & 12 & 13 & 14 & 15 & 16 \\
\hline 1 & 2 & 3 & 4 & 5 & 6 & 23 & 24 & 25 & 26 & 27 & 28 & 29 & 30 & 31 & 32 \\
\hline 1 & 2 & 3 & 4 & 5 & 6 & 7 & 24 & 25 & 26 & 27 & 28 & 29 & 30 & 31 & 32 \\
\hline 17 & 18 & 19 & 20 & 21 & 22 & 23 & 24 & 9 & 10 & 11 & 12 & 13 & 14 & 15 & 16 \\
\hline 17 & 18 & 19 & 20 & 21 & 22 & 23 & 24 & 25 & 10 & 11 & 12 & 13 & 14 & 15 & 16 \\
\hline 1 & 2 & 3 & 4 & 5 & 6 & 7 & 8 & 9 & 10 & 27 & 28 & 29 & 30 & 31 & 32 \\
\hline 1 & 2 & 3 & 4 & 5 & 6 & 7 & 8 & 9 & 10 & 11 & 28 & 29 & 30 & 31 & 32 \\
\hline 17 & 18 & 19 & 20 & 21 & 22 & 23 & 24 & 25 & 26 & 27 & 28 & 13 & 14 & 15 & 16 \\
\hline 17 & 18 & 19 & 20 & 21 & 22 & 23 & 24 & 25 & 26 & 27 & 28 & 29 & 14 & 15 & 16 \\
\hline 1 & 2 & 3 & 4 & 5 & 6 & 7 & 8 & 9 & $\begin{array}{l}10 \\
10\end{array}$ & 11 & 12 & 13 & 14 & 31 & 32 \\
\hline 1 & 2 & 3 & 4 & 5 & 6 & 7 & 8 & 9 & 10 & 11 & 12 & 13 & 14 & 15 & 32 \\
\hline 17 & 18 & 19 & 20 & 21 & 22 & 23 & 24 & 25 & 26 & 27 & 28 & 29 & 30 & 31 & 32 \\
\hline 1 & 18 & 19 & 20 & 21 & 22 & 23 & 24 & 25 & 26 & 27 & 28 & 29 & 30 & 31 & 32 \\
\hline 17 & 18 & 3 & 4 & 5 & 6 & 7 & 8 & 9 & 10 & 11 & 12 & 13 & 14 & 15 & 16 \\
\hline 17 & 18 & 19 & 4 & 5 & 6 & 7 & 8 & 9 & 10 & 11 & 12 & 13 & 14 & 15 & 16 \\
\hline 1 & 2 & 3 & 4 & 21 & 22 & 23 & 24 & 25 & 26 & 27 & 28 & 29 & 30 & 31 & 32 \\
\hline 1 & 2 & 3 & 4 & 5 & 22 & 23 & 24 & 25 & 26 & 27 & 28 & 29 & 30 & 31 & 32 \\
\hline 17 & 18 & 19 & 20 & 21 & 22 & 7 & 8 & 9 & 10 & 11 & 12 & 13 & 14 & 15 & 16 \\
\hline 17 & 18 & 19 & 20 & 21 & 22 & 23 & 8 & 9 & 10 & 11 & 12 & 13 & 14 & 15 & 16 \\
\hline 1 & 2 & 3 & 4 & 5 & 6 & 7 & 8 & 25 & 26 & 27 & 28 & 29 & 30 & 31 & 32 \\
\hline 1 & 2 & 3 & 4 & 5 & 6 & 7 & 8 & 9 & 26 & 27 & 28 & 29 & 30 & 31 & 32 \\
\hline 17 & 18 & 19 & 20 & 21 & 22 & 23 & 24 & 25 & 26 & 11 & 12 & 13 & 14 & 15 & 16 \\
\hline 17 & 18 & 19 & 20 & 21 & 22 & 23 & 24 & 25 & 26 & 27 & 12 & 13 & 14 & 15 & 16 \\
\hline 1 & 2 & 3 & 4 & 5 & 6 & 7 & 8 & 9 & 10 & 11 & 12 & 29 & 30 & 31 & 32 \\
\hline 1 & 2 & 3 & 4 & 5 & 6 & 7 & 8 & 9 & 10 & 11 & 12 & 13 & 30 & 31 & 32 \\
\hline 17 & 18 & 19 & 20 & 21 & 22 & 23 & 24 & 25 & 26 & 27 & 28 & 29 & 30 & 15 & 16 \\
\hline 17 & 18 & 19 & 20 & 21 & 22 & 23 & 24 & 25 & 26 & 27 & 28 & 29 & 30 & 31 & 16 \\
\hline
\end{tabular}

Fig. 2. Frequency sequences for the inquiring device

is much slower than that of the inquiring device. The frequency of each scanning device, known as its phase, cycles through the 32 frequencies in order, according to the value of its clock and changes every $1.28 \mathrm{~s}$.

The scanning device listens continuously on its current frequency during an inquiry scan window of $11.25 \mathrm{~ms}$, long enough for the inquiring device to transmit on an entire train of 16 frequencies. The scanning device then sleeps, before scanning again. This process is repeated periodically. There is some flexibility in the specification [3] as to the length of this period. For our purposes, we have chosen the value $0.64 \mathrm{~s}$.

If the scanning device successfully hears a message, by listening on the right frequency at the right time (when the inquiring device is transmitting a packet), it will switch to the inquiry response substate, in which it waits 2 time slots (i.e. $625 \mu \mathrm{s}$ ) and then sends a reply on the same frequency. A contention problem arises when two devices in inquiry scan try to reply to the same inquiry packet. In this case, the two replies collide and are both lost. To avoid repetition of such a problem, after sending a reply, a device draws a random number $N \in[0, \ldots, 127]$ and waits for $2 \cdot N$ time slots before going back to the inquiry scan substate. Note that the maximum random wait is sometimes higher than 127 but, according to the specification [3, this is an appropriate value for our scan period of $0.64 \mathrm{~s}$. After each successful receive, the scanning device also adds one to its phase. Figure 3 summarises the steps of the overall process and the time spent in each.

\section{Modelling in PRISM}

From the description in the previous section, it should be clear that the performance of the Bluetooth inquiry process, i.e. the time required for messages 


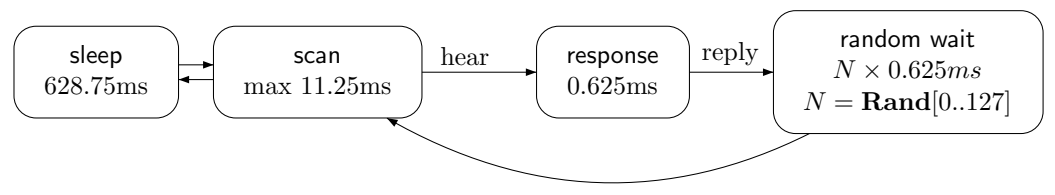

Fig. 3. Timing of the scanning device's behaviour

to be successfully sent and received, is the result of the interaction between two non-trivial sequences of events. This motivates the need for the construction and analysis of a formal model. We now describe how this can be achieved using the tool PRISM and its high-level model description language.

We consider a single inquiring device and a single scanning device, which in this section we refer to as the sender and receiver, respectively. The clocks of both devices are digital, whose time is incremented in discrete steps, corresponding to $312.5 \mu$ s slots, and whose drift can be assumed to be negligible during the relative short inquiry process. Since the behaviour of the receiver is probabilistic, the model we construct is a discrete-time Markov chain (DTMC). Note that, as we will discuss later, the model contains no nondeterminism and we can thus avoid using a Markov decision process (MDP).

\subsection{Modelling the Sender (Inquiring Device)}

The behaviour of the sender was described in Section 3.1 and illustrated in Figures 1 and 2. The corresponding PRISM code is shown in Figure 4 In the PRISM language, a model's description comprises a number of modules, each corresponding to a component of the system being modelled. We specify the sender with a single module.

The initial part of a module definition lists a set of finite-ranging variables which determine the possible states that the module can be in. The first variable of the sender module is $s$, which keeps track of which step of the protocol the sender is on: when $s=1$, it sends two sequential messages on a pair of odd/even frequencies; when $s=2$, it scans for a reply on the same two frequencies. The four variables $f, c, o$ and rep keep track of which frequency is currently being used and the position in the frequency sequence of Figure 2. Their exact meaning can be inferred from the comments in Figure 4. The last variable rec counts the number of replies received.

The behaviour of a module is described by a set of guarded commands of the following form:

$$
\text { [action] guard } \rightarrow \text { update; }
$$

The guard is a predicate over the module's variables (and in fact the variables of all other modules). When the guard is satisfied, the behaviour of the module is determined by the update, given in terms of how the values of the module's variable should change. The first guarded command of the sender module, for instance, states that if $s=1$ and $f$ is odd then $f$ is incremented by 1 and, if $f=c$, the value of $o$ is reversed (we use the C-style question mark operator for 


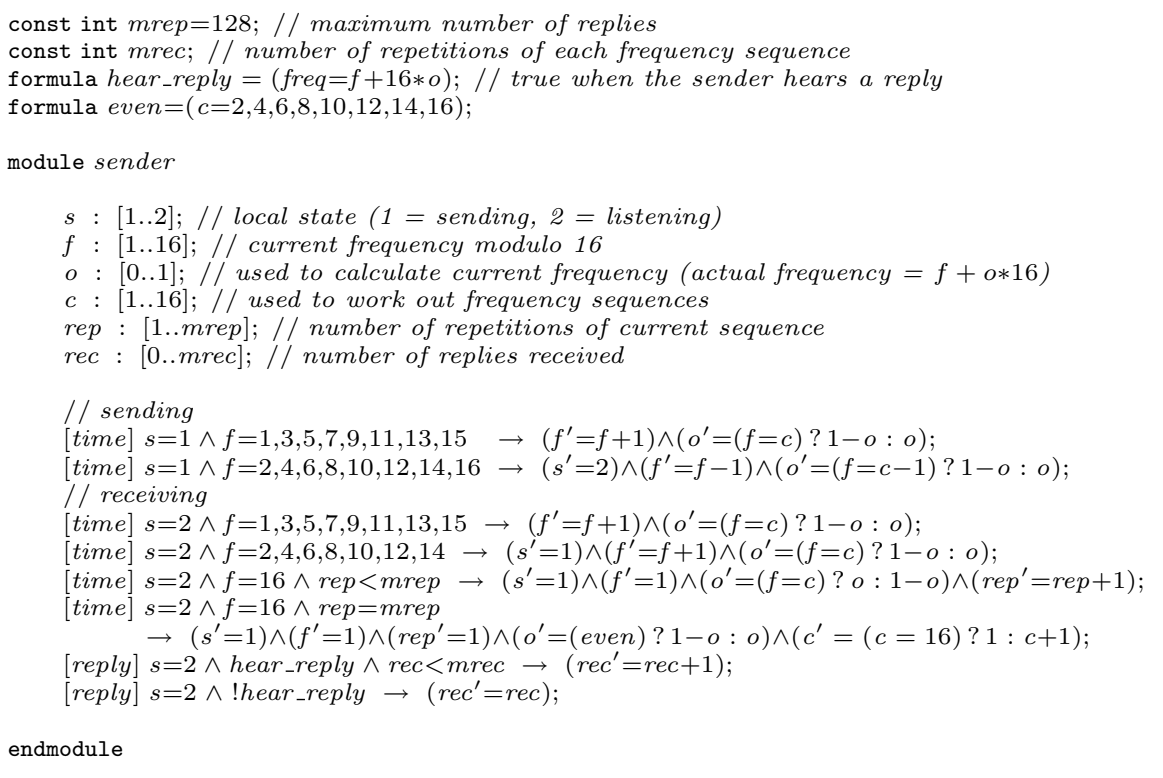

Fig. 4. PRISM module representing the sender

conditional evaluation). This corresponds to the sender finishing transmitting on an odd frequency and changing to the subsequent even frequency.

Contained in the square brackets at the start of each guarded command is an (optional) action label. This is used to synchronise with other modules in the PRISM model. More precisely, in a state of the entire model (which is a parallel composition of all modules), transitions of modules corresponding to guards labelled with identical actions will occur simultaneously in a single global transition. One example is the time action, which labels many of the commands. Since we can assume that Bluetooth devices all operate at the same clock-speed, we model time elapsing in a synchronous fashion. For each $312.5 \mu$ s time slot which passes, all modules synchronise on a time action, with the transition of each module reflecting the changes that occur in that time slot. All other commands (some of which are synchronous, see e.g. the reply action used in Figure 4 and some of which are asynchronous) are assumed to correspond to an instantaneous change in state.

The code also illustrates other features of PRISM such as the use of constants (e.g. mrep), which allow definitions of fixed values to be kept separate (and possibly left undefined until run-time), and formulas (e.g. hear_reply), which allow complex expressions to be defined once and then reused.

\subsection{Modelling the Receiver (Scanning Device)}

The behaviour of a receiver was previously summarised in Figure 3 For convenience, the corresponding PRISM model comprises two modules: receiver (Fig- 
ure 57, the main part of the receiver's behaviour; and receiver_frequency (Figure 6) which keeps track of the frequency that the receiver will use next time it starts a scan (determined by the device's clock).

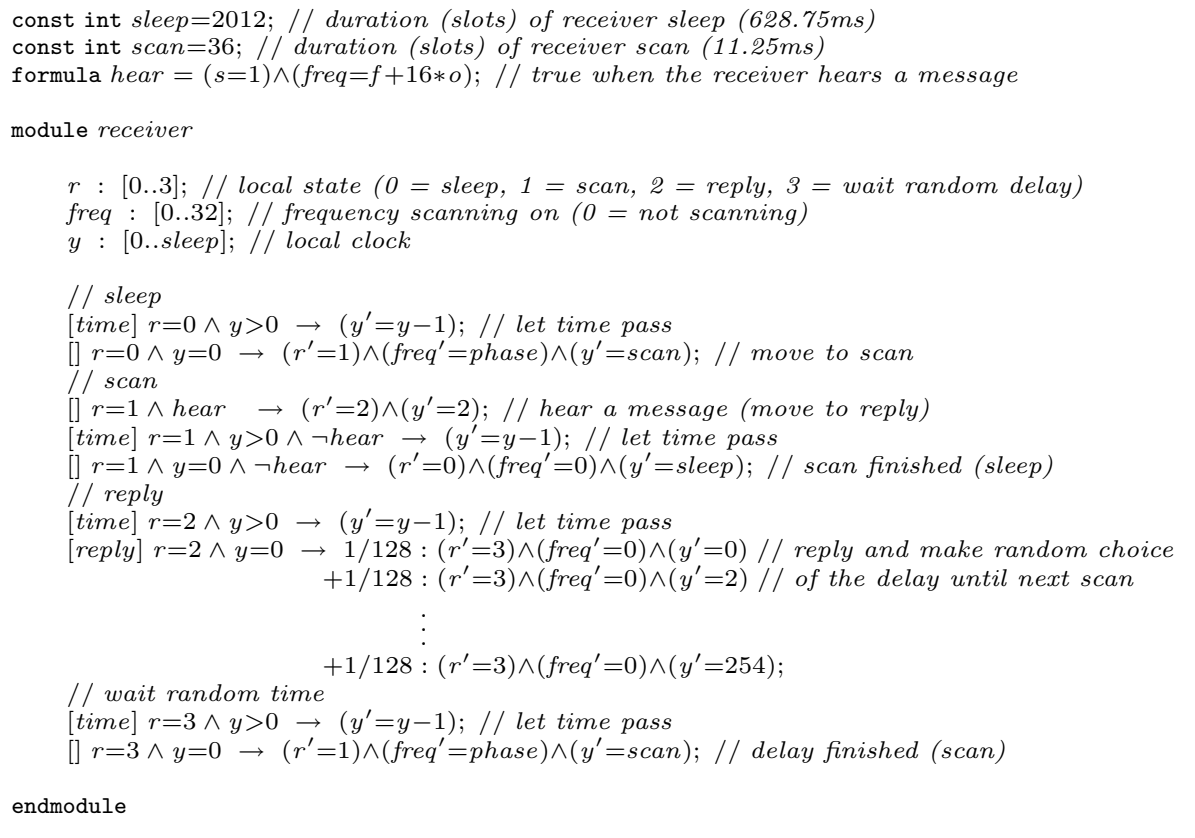

Fig. 5. PRISM module representing the receiver

The format of the guarded command notation used has already been explained in the previous section. One new feature is the method for specifying probabilistic choice. This can be seen in Figure 5 in the command labelled with the guard " $r=2 \wedge y=0$ ". Several possible updates are given, each with an associated probability. Here, this represents the receiver drawing the random number $N$ (see Section 3.2 .

Note that these two modules again have commands labelled with the time and reply actions, which causes all three modules to make these transitions synchronously. Note also that some commands in the receiver module have no action label. This means that they occur independently from the other modules. However, the fact that this is the only module with such commands, combined with the fact that the behaviour of each individual module is always deterministic (i.e. all of its guards are disjoint), means that the overall model contains no nondeterminism, and is hence suitable for representation as a DTMC. 


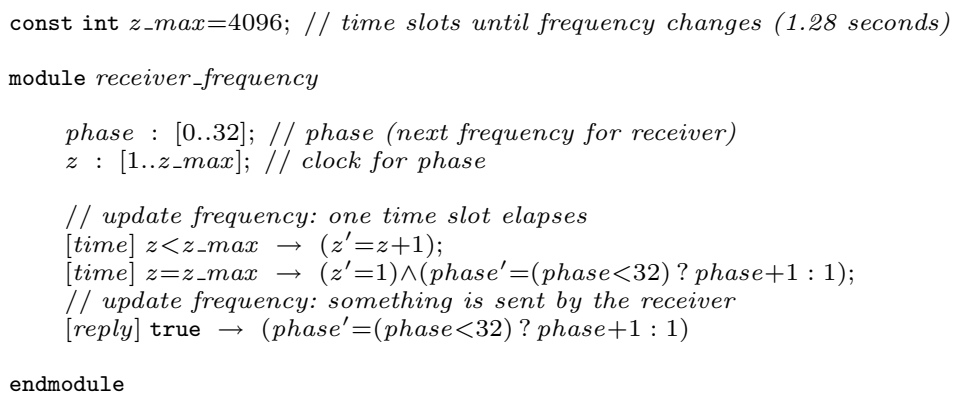

Fig. 6. PRISM module that computes the phase of the receiver

\subsection{Reducing Model Complexity}

The PRISM code in Figures 46 is intended to provide a clear description of our model. In fact, we have made a number of subsequent optimisations, which we describe in this section 11 The changes made provide an extremely useful increase in efficiency. It is worth noting that one simplification we could not perform was to reduce the model by scaling down some of the large constants for lengths of delays and cycles. This is because important events also occur at the level of individual time slots.

The first and most effective optimisation we performed is based on the fact that, when there is only one receiver, and when this receiver enters its sleep state, the behaviour of the whole system is totally deterministic: the receiver will come out of sleep exactly 2012 time units later. The corresponding shift of the sender along its sequence of frequencies in this period and the change in phase of the receiver are relatively easy to compute. We can thus compact all 2012 transitions of this sleeping process into a single transition. This reduces the maximum value of the clock $y$ from 2012 to 254 (maximum random delay).

In fact, we can extend this optimisation. At the beginning of a scan, it is possible to determine, from the current state, whether a message will be successfully heard during the scan. In cases where it will not, we can skip the scan and incorporate the 36 time slots that would have been spent into the subsequent 2012 slot sleep transition. Furthermore, this makes the total jump in this case 2048 slots which, being a multiple of the train length, makes computing the sender's next frequency much easier.

Lastly, we note that, because of the regular alternation between send and scan of the sender, and the fact that a receiver always replies to a successfully detected message after exactly two time slots, we can be sure that the replies will always be successfully received by the sender. Hence, the formula hear_reply in Figure 4 and indeed the last guarded command can be removed.

${ }^{1}$ The final version of the code can be found at: www.cs.bham.ac.uk/ \{\}dxp/prism/casestudies/bluetooth.html 


\section{Experimental Results}

Our primary concern is the performance of the Bluetooth inquiry process, i.e. the time required for a master device to successfully receive replies from listening slave devices. This is affected by the number of times the receiver sleeps before successfully scanning the right frequency at the right time, and the random delays selected. More specifically, since the protocol is probabilistic in nature, we compute the expected time for the inquiry process to complete, with completion occurring when the number of replies received reaches a predetermined bound.

We performed an analysis of the model described in the previous section with a prototype extension of the PRISM 2.0 model checker, which allows us to assign costs to states and transitions of the model and then compute, for example, the expected cumulated cost before reaching some set of states. In our model the cost we measure is time. We assign a cost of 1 to all time actions and 0 to all others ${ }^{2}$ Our experiments were performed on a $1 \mathrm{~GB} 2.80 \mathrm{GHz}$ workstation.

The first issue we must address is the initial configuration of the model. We cannot assume that the sender and receiver both start in some fixed state because this is unrealistic. For efficiency reasons, we restrict ourselves to the case where the sender is already transmitting inquiry packets and a receiver begins scanning after some unknown delay, which is a reasonable scenario. We can hence fix the initial state of the receiver (i.e. variables $r$, freq, and $y$ ). We can also fix $r e c=0$ in the sender module since this cannot increase until after the receiver begins scanning. Note that we cannot fix the actual phase of the receiver since this is determined by its clock, whose value could be anything when it first begins scanning. This leaves us with $2 \cdot 16 \cdot 2 \cdot 16 \cdot 128 \cdot 4096 \cdot 32=17,179,869,184$ possible initial states.

Since formal verification aims to be exhaustive, we must consider all of these, although clearly it is not feasible to treat each one separately. Fortunately, we can deal with this in PRISM by building a single DTMC with multiple initial states and then examining the results of model checking for all these states ${ }^{3}$ Initially, this single model proved too large for the workstation we were using. However, by partitioning the set of all initial states into classes (we fix variable phase, giving 32 sets of $536,870,912$ states each), we reduce the problem to 32 separate instances of model checking, each of which was feasible.

As we will see from the results in the following paragraphs, though, despite this partitioning (and despite the reductions in model complexity described in Section 4.3, this still results in models with extremely large state spaces. Intuitively, this is because the Bluetooth devices execute complicated sequences of events, comprising both actions that take a large number of time-slots and those performed in a single slot. Fortunately, there remains a certain degree of regularity in the process and we are able to exploit this using PRISM's sym-

\footnotetext{
${ }^{2}$ As described in Section 4.3. we actually sometimes accumulate multiple time steps into a single step. The costs of these transitions are modified accordingly.

${ }^{3}$ In fact, this required a modification to PRISM, but only a trivial one: the tool always computes results for all states in the model anyway.
} 
bolic (BDD-based) implementation. With an approach based on explicit data structures (e.g. sparse matrices) this would not be feasible.

\subsection{Time for a Single Reply}

We first present results for the time required for the receiver to successfully send a single reply. In fact, since the receiver does not make a random choice until after it first replies to a message, the expected time computed is in this case the exact (deterministic) time required. The 32 models we constructed each contained approximately $3.4 \times 10^{9}$ states, required less than a minute to construct, and took 1-2 seconds to analyse.

We hence computed the expected time to send a message for all possible initial configurations. Using PRISM, we were able to extract information about the best and worst case scenarios. The minimum time is $635 \mu \mathrm{s}$ ( 2 slots), which corresponds to the cases when the receiver starts listening on the frequency that the sender is currently sending messages on, and therefore the receiver sends a reply after waiting 2 slots. The maximum time is $2.5716 \mathrm{~s}(8,229$ slots $)$ and is achieved in 860,160 of the possible initial states. This maximum time results when the receiver does not hear the sender until it scans for the fifth time and therefore sleeps 4 times. We now give an example execution which illustrates exactly how this can arise.

Example 1. Suppose that the receiver starts its first scan on frequency 1 and that its phase is about to change. Suppose also that the sender is performing its last repetition of the first of the following frequency sequences:

$\begin{array}{cccccccccccccccc}1 & 18 & 19 & 20 & 21 & 22 & 23 & 24 & 25 & 26 & 27 & 28 & 29 & 30 & 31 & 32 \\ 17 & 18 & 3 & 4 & 5 & 6 & 7 & 8 & 9 & 10 & 11 & 12 & 13 & 14 & 15 & 16 \\ 17 & 18 & 19 & 4 & 5 & 6 & 7 & 8 & 9 & 10 & 11 & 12 & 13 & 14 & 15 & 16 \\ 1 & 2 & 3 & 4 & 21 & 22 & 23 & 24 & 25 & 26 & 27 & 28 & 29 & 30 & 31 & 32\end{array}$

and has already sent on frequency 1 during this repetition. During this scan, the sender will finish the sequence and start the following one which does not contain 1. The receiver will not hear anything during this scan and will therefore enter sleep. When the receiver wakes, the sender will still be using the second frequency sequence and the receiver will now be scanning on frequency 2 (as its phase was about to change when it first scanned), and therefore the receiver will not hear anything and sleep again. In fact, since the sender's subsequent frequency sequence does not contain 2 or 3 , the receiver will not hear anything on either of these two sequences as, during this time, the receiver will be scanning on 1, 2 or 3 . Only when the receiver wakes for the fourth time will its scan be successful on frequency 3 because the sender will start using the fourth sequence.

In Figure 7 (a) we have plotted the time until the sender hears a reply against the number of initial states that result in this time. The discontinuities in the graph are to be expected and follow from the fact that, when the receiver does not hear anything during a scan (36 time slots), it sleeps for 2,012 slots before scanning again. Since, in the worst case, the receiver sleeps 4 times before hearing something from the sender, there are 5 peaks in the graph corresponding to the 


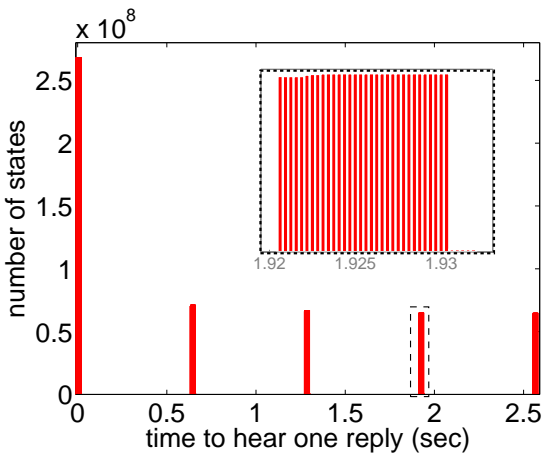

(a) distribution over initial states

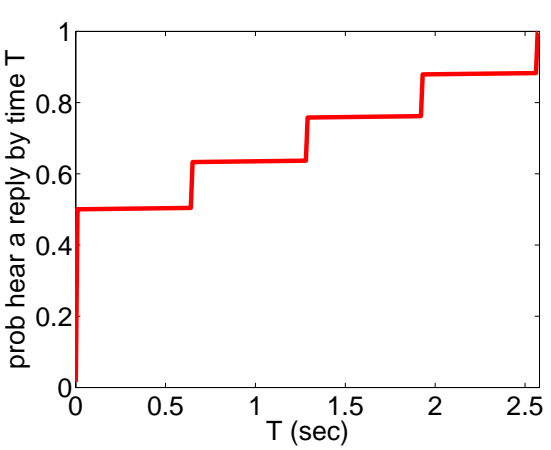

(b) cumulative distribution

Fig. 7. Time for the sender to hear a reply from the receiver

\begin{tabular}{|c|c|c|c|c|}
\hline$K$ & $n=1$ & $n=1(\mathrm{v} .1 .1)$ & $n=2$ & $n=2$ (derived) \\
\hline 0 & 0.500305 & 0.461240 & 0.455379 & 0.250305 \\
1 & 0.633575 & 0.596265 & 0.590829 & 0.383657 \\
2 & 0.759062 & 0.731585 & 0.728684 & 0.526981 \\
3 & 0.879674 & 0.857913 & 0.855329 & 0.681114 \\
4 & 1 & 0.984295 & 0.984218 & 0.849408 \\
5 & 1 & 0.988269 & 0.988294 & 0.911750 \\
6 & 1 & 0.992398 & 0.992514 & 0.956496 \\
7 & 1 & 0.996294 & 0.996519 & 0.985521 \\
8 & 1 & 1 & 1 & 1 \\
\hline
\end{tabular}

Table 1. Probability of sleeping $K$ times before sending $n$ replies

receiver sleeping from 0 up to 4 times. The inset in Figure 7(a) illustrates one of these peaks more clearly. The width of each peak is $11.25 \mathrm{~ms}$ (36 slots).

If we make the assumption that, when the receiver first starts to listen, there is a uniform distribution on the set of possible initial configurations, we can calculate the cumulative probability distribution function for the time for the sender to hear a reply (see Figure $7(\mathrm{~b})$ ). Furthermore, from this distribution, Column 2 of Table 1 shows the probability that the receiver sleeps at most $K$ times before sending its first reply to the sender.

\subsection{Time for Two Replies}

For the case where the sender waits until two replies have been received, the 32 constructed models each have approximately $5.6 \times 10^{10}$ states and took roughly 80 minutes to build and 165 minutes to model check. The minimum expected time, over all possible initial configurations, for the sender to hear two replies is $0.0456 \mathrm{~s}$ (146.0 slots). The maximum is 5.177 seconds (16,565 slots) and 518 
of the possible initial states result in this. This is possible since the receiver can sleep up to 8 times before sending its second reply. We now extend Example 1 to illustrate how such a scenario can occur.

Example 2. Example 1 demonstrated the receiver sleeping 4 times and then finally hearing the sender when it is on frequency 3 in the sequence:

$\begin{array}{llllllllllllllll}1 & 2 & 3 & 4 & 21 & 22 & 23 & 24 & 25 & 26 & 27 & 28 & 29 & 30 & 31 & 32\end{array}$

After the receiver scans, it increases its phase by 1 and waits a random delay before scanning again. During this random delay the phase will increase again and the receiver will next scan on frequency 5 . Since this frequency does not appear on the above sequence and the sender has just started using it, he will not hear anything during this scan and the next one (still using this sequence), and will therefore sleep twice. When the receiver wakes again he will scan on frequency 6 and since the sender's subsequent frequency sequences will be:

$\begin{array}{cccccccccccccccc}1 & 2 & 3 & 4 & 5 & 22 & 23 & 24 & 25 & 26 & 27 & 28 & 29 & 30 & 31 & 32 \\ 17 & 18 & 19 & 20 & 21 & 22 & 7 & 8 & 9 & 10 & 11 & 12 & 13 & 14 & 15 & 16\end{array}$

it follows that the receiver will sleep an additional 2 times before finally hearing (for the second time) from the sender while scanning on frequency 7 .

In Figure 8 we have plotted, for the expected time for the receiver to reply to two messages, both the distribution over the states and the cumulative distribution function (where, as in Section 5.1, in this case we assume that there is a uniform probabilistic choice as to the state of the system when the receiver first scans). As in Figure 7, the discontinuities are due to the time that the receiver spends in sleep: in Figure 8(a) there are 9 peaks - the last 4 being considerably smaller than the first 5 but still visible - which correspond to the cases when the receiver sleeps from 0 to 8 times before sending its second reply. The inset in this case illustrates that, for some initial states, the expected time is not included within these peaks.

In Figure 8 (b) we have also included a second cumulative distribution function, derived from the earlier distribution of Figure 7(b), under the assumption that the time to hear the second message is independent of the time to hear the first message. This derivation is obtained by taking the convolution of two copies of the distribution in Figure 7(b) together with a distribution representing the random delay made by the receiver between sending the first reply and beginning its next scan. The rightmost two columns of Table 1 show the probabilities, extracted from these graphs, of sleeping at most $K$ times before sending two replies. Interestingly, these results demonstrate that the assumption that the time to reply to the second message is independent of the time to reply to the first is incorrect, i.e. leads to inaccurate results. More precisely, the results show that if the receiver sleeps before sending its first reply, it is less likely to sleep the second time.

We have also attempted to compute probabilities for higher numbers of replies. However, in these cases, partitioning into 32 models is not feasible. We have been able to generate results for up to 5 replies by partitioning the initial states more finely, but this means that the total number of verifications required grows considerably and hence we have not completed an exhaustive analysis. 


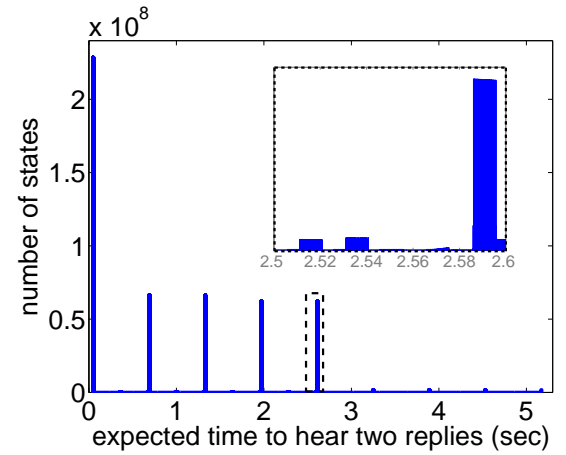

(a) distribution over initial states

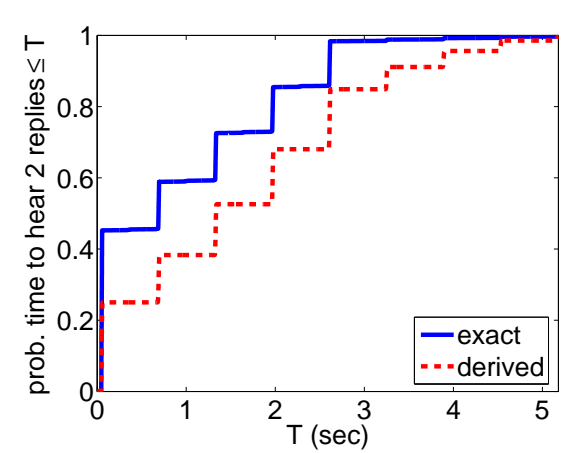

(b) cumulative distribution

Fig. 8. Time for the sender to hear two replies from the receiver

\subsection{Comparison with Bluetooth Version 1.1}

Finally, we have also carried out a comparison of the most recent version (1.2) of the Bluetooth specification (as described so far in this paper) and the previous version 1.1. The main difference in terms of the protocol is that, in version 1.1, the receiver only sends replies to every second message received. We modified our model to reflect these changes and recomputed the expected time for the inquiry process. The results can be found in Table 1. Unsurprisingly, we see that the time for one reply in version 1.1 and for two replies in version 1.2 are very similar. However, we successfully illustrate that, as was intended, version 1.2 indeed results in improved expected times.

\section{Related Work}

Thanks to the ongoing growth in popularity of Bluetooth technology, an increasing amount of research is being carried out in order to analyse and improve its efficiency. There is, however, a limited amount of work regarding the inquiry process of the protocol. To our knowledge, this paper is the first application of formal verification to the area: the most common form of analysis being used is simulation, with tools such as ns-2 [4] and BlueHoc [1]; see for example [115].

Elsewhere, attempts have also been made to compute the time required for inquiry analytically. Two examples include [10] and [12. In the former, the emphasis is primarily on the issue of scatternet formation, but they also discuss a symmetric variation of the Bluetooth inquiry process, and consider analytic expressions for the completion time, comparing them to that of the standard asymmetric version. The latter considers the standard version, but for an arbitrary number of devices, rather than just two. The authors also complement this with results from a discrete-event simulation. Both papers, however, take a far more simplistic approach to modelling the inquiry process than us. Firstly, 
they assume that the sender uses a single train of 32 frequencies which remains constant throughout, drastically reducing the complexity of its behaviour. They therefore also assume that the receiver will always be able to listen to all frequencies in a short period, and thus never need to sleep. Hence, in their analysis, they take the frequency synchronisation delay to be uniformly distributed.

A more comprehensive analysis was recently presented in 8 . In similar fashion, the authors produce an analytic expression for the probability distribution function of the time to complete an inquiry and then use this to validate a discrete-event simulation of the same model. Like us, they consider the correct behaviour in terms of trains (although some simplifications are still required in order to derive an analytic expression), and hence the model is much closer to ours. The most significant difference is that whereas we aim for an exact/worstcase analysis, considering all possible overlaps between the sender and receiver, they assume an equiprobable distribution between these in order to derive a probability function. This, combined with the fact that they use different parameters to those used here (both are compliant with the official specification), means that a detailed comparison of the two sets of results is impractical. Unfortunately, the publication describing the derivation of the distribution function is not yet available. In future, we plan to carry out a more comprehensive comparison of this work with our own.

It is also worth noting that all of the related work mentioned above is based on either version 1.0 or 1.1 of the specification. In this paper, we focus on the most up-to-date version, 1.2.

\section{Discussion and Conclusion}

In this paper, we have presented a formal analysis of the performance of Bluetooth device discovery, using probabilistic model checking and the tool PRISM. We showed how this permits an exhaustive analysis of a low-level model of the specification. This allows us to examine the best and worst case expected times for the inquiry process and identify exactly how these situations can occur.

We are, however, limited to a certain extent by the huge size of the probabilistic models that we need for this process. Techniques based on discrete-event simulation are far less susceptible to this phenomenon, but have two disadvantages: firstly, they compute only approximations to the numerical results we obtain; and secondly, they sometimes require additional probabilistic assumptions (in this case study, on the initial configuration of the Bluetooth devices). It would be advantageous to compare or even combine the two approaches. One interesting application of the results in this paper might be to use our exact results to improve the accuracy of a simulation of Bluetooth performed at a higher level. We plan to investigate this area further.

There are also several other directions in which we we would like to extend this work. Two examples are: increasing the number of messages received and increasing the number of receivers. The latter introduces several new dimensions such as collision of messages between devices and tracking which replies 
correspond to which receivers. We would also like to address power consumption issues (which are particularly relevant since Bluetooth is aimed at portable low-power devices where battery life is crucial). This can be achieved using the existing support for cost-based analysis in PRISM. Lastly, it would be interesting to study the effect of noise and/or interference on the inquiry procedure. Since all of these areas lead to an increase in model size, we will almost certainly need to consider additional techniques, such as combination with simulation (as discussed above) or abstraction and symmetry reduction methods.

\section{References}

1. BlueHoc - An open-source Bluetooth simulator: wWw-124.ibm.com/developerworks/opensource/bluehoc

2. PRISM web site. www.cs.bham.ac.uk/ ${ }^{\mathrm{d} x p / p r i s m . ~}$

3. Specification of the Bluetooth system, version 1.2, Bluetooth SIG, 2003, www. bluetooth.com

4. The Network Simulator - ns-2: www.isi.edu/nsmam/ns

5. Basagni, S., Bruno, R., and Petrioli, C. Device discovery in Bluetooth networks: A scatternet perspective. In Proc. Networking 2002, volume 2345 of LNCS, pages 1087-1092. Springer, 2002.

6. Kasten, O. and Langheinrich, M. First experiences with Bluetooth in the Smart-Its distributed sensor network. In Proc. PACT'01, 2001.

7. Kwiatkowska, M., Norman, G., and Parker, D. PRISM 2.0: A tool for probabilistic model checking. In Proc. 1st International Conference on Quantitative Evaluation of Systems (QEST'04), 2004. To appear.

8. Peterson, B., Baldwin, R., and Kharoufeh, J. A specification-compatible Bluetooth inquiry simplification. In Proc. Hawaii Int. Conference on System Sciences (HICSS'04), 2004.

9. Rutten, J., Kwiatkowska, M., Norman, G., and Parker, D. Mathematical Techniques for Analyzing Concurrent and Probabilistic Systems, volume 23 of CRM Monograph Series. American Mathematical Society, 2004.

10. Salonidis, T., Bhagwat, P., Tassiulas, L., and LaMaire, R. Proximity awareness and ad hoc network establishment in Bluetooth. Technical Report TR 2001-10, Institute of Systems Research, University of Maryland, 2001.

11. Siegemund, F. and Rohs, M. Rendezvous layer protocols for Bluetooth-enabled smart devices. In Proc. Conference on Architecture of Computing Systems - Trends in Network and Pervasive Computing (ARCS'02). Springer, 2002.

12. Záruba, G. and Gupta, V. Simplified Bluetooth device discovery - Analysis and simulation. In Proc. Hawaii Int. Conference on System Sciences (HICSS'04), 2004. 